\title{
QCD flux tubes across the deconfinement phase transition
}

\author{
Paolo Cea ${ }^{1,2}$, Leonardo Cosmai ${ }^{1, \star}$, Francesca Cuteri ${ }^{3}$, and Alessandro Papa ${ }^{4}$ \\ ${ }^{1}$ INFN - Sezione di Bari, I-70126 Bari, Italy \\ ${ }^{2}$ Dipartimento di Fisica dell'Università di Bari, I-70126 Bari, Italy \\ ${ }^{3}$ Institut für Theoretische Physik, Goethe Universität, 60438 Frankfurt am Main, Germany \\ ${ }^{4}$ Dipartimento di Fisica, Università della Calabria, \& INFN - Gruppo Collegato di Cosenza, I-87036 Rende, \\ Italy
}

\begin{abstract}
We study the behavior across the deconfinement phase transition of the chromoelectric flux tube generated by a static quark and a static antiquark for several distances between them. We present preliminary results for distances up to $1.33 \mathrm{fm}$ and temperatures up to $1.5 T_{c}$.
\end{abstract}

\section{Introduction}

Lattice formulation of gauge theories allows us to investigate the color confinement phenomenon within a nonperturbative framework. Indeed, Monte Carlo simulations produce samples of vacuum configurations that, in principle, contain all the relevant information on the nonperturbative sector of QCD. A wealth of numerical analyses in QCD has firmly established that the chromoelectric field between a static quark-antiquark pair distributes in tubelike structures or "flux tubes" [1-20]. From these tubelike structures a linear potential between static color charges naturally arises, thus representing a numerical evidence of color confinement.

In our recent studies color flux tubes dominantly made up of chromoelectric field directed along the line joining a static quark-antiquark pair have been investigated, in the cases of zero temperature [14, $15,21]$ and nonzero temperature [22]. In the present paper we present new results obtained in studying the flux tubes across the deconfinement phase transition. In particular we show preliminary results for several distances between the static quark-antiquark sources and temperatures up to $1.5 T_{c}$. The plan of the presentation is as follows: in section 2 we discuss the observables needed to extract the field strength tensor of the static quark-antiquark sources; in section 3 we present our numerical results; and in section 4 our conclusions.

\section{Lattice observables and numerical setup}

The field distributions generated by a static quark-antiquark pair can be probed by calculating on the lattice the vacuum expectation value of the following connected correlation function $[5,23]$ :

$$
\rho_{W, \mu \nu}^{\text {conn }}=\frac{\left\langle\operatorname{tr}\left(W L U_{P} L^{\dagger}\right)\right\rangle}{\langle\operatorname{tr}(W)\rangle}-\frac{1}{N} \frac{\left\langle\operatorname{tr}\left(U_{P}\right) \operatorname{tr}(W)\right\rangle}{\langle\operatorname{tr}(W)\rangle} .
$$

${ }^{\star}$ Speaker, e-mail: leonardo.cosmai@ba.infn.it 


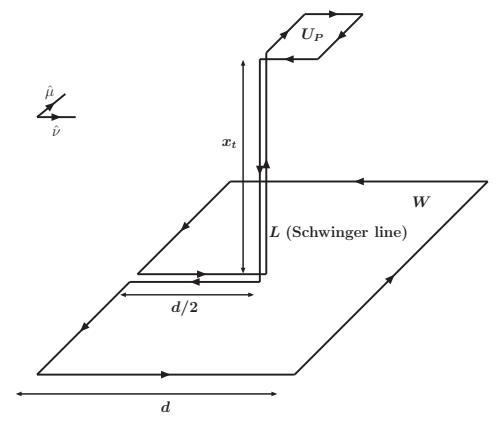

(a)

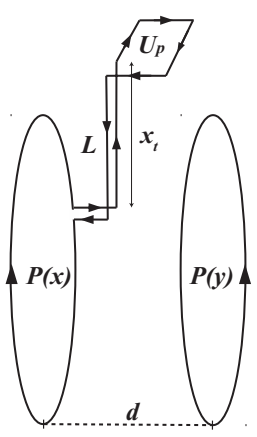

(b)

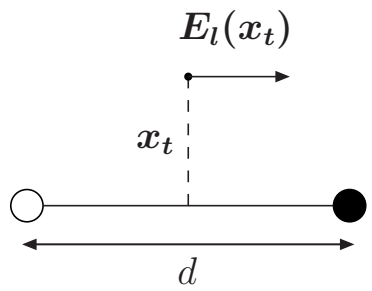

(c)

Figure 1. (a) The connected correlator given in Eq. (1) between the plaquette $U_{P}$ and the Wilson loop (subtraction

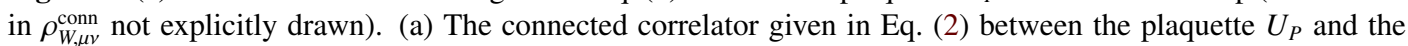
Polyakov loop (subtraction in $\rho_{P, \mu v}^{\text {conn }}$ not explicitly drawn). (c) The longitudinal chromoelectric field $E_{l}\left(x_{t}\right)$ with respect to the position of the static sources (represented by the white and black circles), for a given value of the transverse distance $x_{t}$.

Here $U_{P}=U_{\mu \nu}(x)$ is the plaquette in the $(\mu, \nu)$ plane, connected to the Wilson loop $W$, lying on the $\hat{4} \hat{i}$-plane, with $\hat{i}$ any fixed spatial direction, by a Schwinger line $L$, and $N$ is the number of colors (see Fig. 1(a)). In the case of nonzero temperature the role of the Wilson loop is played by two Polyakov loops (see Fig. 1(b)):

$$
\rho_{P, \mu \nu}^{\text {conn }}=\frac{\left\langle\operatorname{tr}\left(P(x) L U_{P} L^{\dagger}\right) \operatorname{tr} P^{\dagger}(y)\right\rangle}{\left\langle\operatorname{tr}(P(x)) \operatorname{tr}\left(P^{\dagger}(y)\right)\right\rangle}-\frac{1}{3} \frac{\left\langle\operatorname{tr}(P(x)) \operatorname{tr}\left(P^{\dagger}(y)\right) \operatorname{tr}\left(U_{P}\right)\right\rangle}{\left\langle\operatorname{tr}(P(x)) \operatorname{tr}\left(P^{\dagger}(y)\right)\right\rangle},
$$

where the two Polyakov lines are separated by a distance $d$. The quark-antiquark field strength tensor is obtained as (for a discussion see Ref. [21])

$$
F_{\mu v}(x)=\frac{1}{a^{2} g} \rho_{W, P, \mu v}^{\mathrm{conn}}(x) .
$$

By varying the orientation of the plaquette $U_{P}$ (Fig. 1(a), 1(b)) it is possible to evaluate all the components of the chromoelectromagnetic tensor. We measure the field on the locus of points that are equidistant from the two sources, with $x_{t}$ measuring the distance between the point of measure and the intersection of the above mentioned locus of points with the $1 d$ axis connecting the static sources (Fig. 1(c)). The numerical results presented here refer to different values of $x_{t}$ and several choices of the distance $d$ between the static sources. The role of the distance $d$ between the static sources has been discussed in Ref. [24].

We performed numerical simulations for pure gauge $\mathrm{SU}(3)$ on $40^{3} \times 10$ and $48^{3} \times 12$ lattices, and temperatures in the range $0.8 T_{c} \leq T \leq 1.5 T_{c}$. The typical statistics of each run consisted of about 4-5 thousands of configurations; to allow for thermalization we typically discarded a few thousand sweeps. The lattice discretization that we used for the pure gauge SU(3) is the standard Wilson action, with the physical scale set assuming for the string tension the standard value of $\sqrt{\sigma}=420 \mathrm{MeV}$ and using the parameterizationgiven in [25]. For all simulations we made use of the publicly available MILC code [26], suitably modified in order to introduce the relevant observable. 


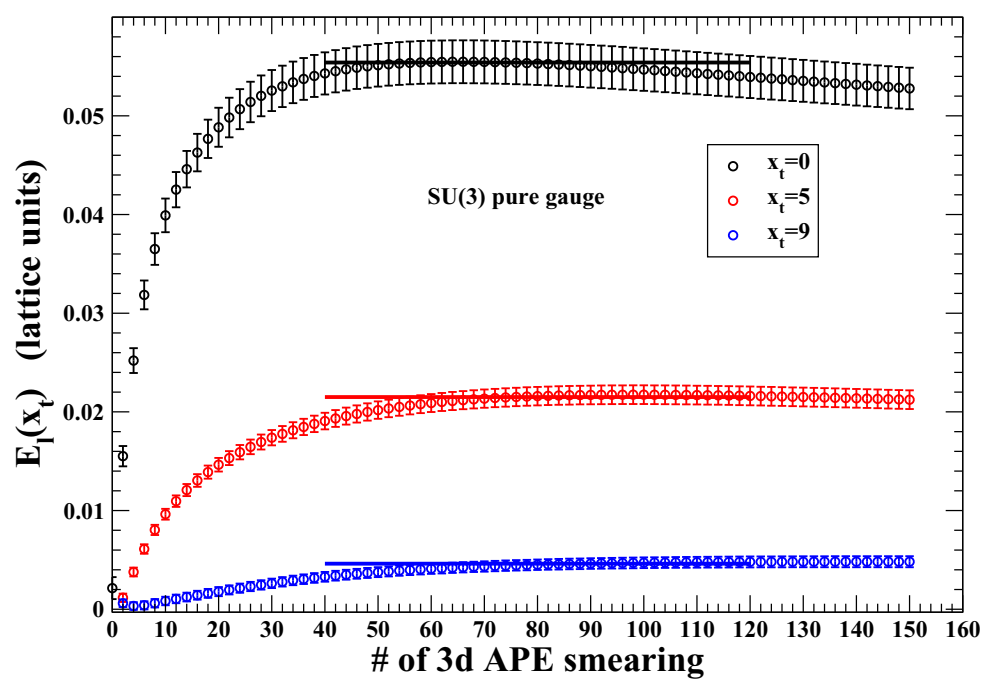

Figure 2. Behavior of the longitudinal chromoelectric field $E_{l}$, on a given lattice and for various values of the distance from the axis connecting the static sources, versus the number of APE smearing steps on the spatial links.

\section{Numerical results}

\subsection{Smoothing procedure}

The connected correlator defined in Eq. (1) suffers from large fluctuations at the scale of the lattice spacing, which are responsible for a bad signal-to-noise ratio. To extract the physical information carried by fluctuations at the physical scale (and, therefore, at large distances in lattice units) we smoothed out configurations by the smearing procedure. Our setup consisted of (just) one step of HYP smearing [27] on the temporal links, with smearing parameters $\left(\alpha_{1}, \alpha_{2}, \alpha_{3}\right)=(1.0,0.5,0.5)$, and $N_{\mathrm{APE}}$ steps of APE smearing [28] on the spatial links, with smearing parameter $\alpha_{\mathrm{APE}}=0.167$. Here $\alpha_{\mathrm{APE}}$ is the ratio between the weight of one staple and the weight of the original link. The optimal number of smearing steps was found by looking at the smearing step at which our direct observable $E_{l}\left(x_{t}\right)$ showed the largest signal-to-noise ratio, with the smearing parameter tuned in such a way that in the $E_{l}\left(x_{t}\right)$ versus 'smearing step' plot we could see a clear plateau.

In Fig. 2 we show the behavior under smearing of the longitudinal chromoelectric field $E_{l}\left(x_{t}\right)$ on a $40^{3} \times 10$ lattice at $\beta=6.050$ and quark-antiquark distance $d=12 a$ ( $a$ is the lattice spacing) corresponding to a physical distance $d=1.14 \mathrm{fm}$. We can see that, for each value of the distance $x_{t}$ in the direction transverse to the axis connecting the sources, a clear plateau is reached after a sufficiently large number of smearing steps. All results concerning the chromoelectric field $E_{l}\left(x_{t}\right)$ presented in the following will always refer to determinations on smeared configurations, after a number of smearing steps $N_{\mathrm{APE}}$ such that the plateau is reached for all considered values of $x_{t}$. The typical value of $N_{\mathrm{APE}}$ ranges between 50 and 150 . 


\subsection{Continuum scaling}

Our aim is to determine the physical properties of the chromoelectric flux tube in the continuum, for this reason, we have preliminarily checked that our simulations are performed in a region of values of the coupling $\beta$ where continuum scaling holds. We have hence measured the longitudinal chromoelectric field generated when the static sources are located at the same physical distance $d$, but for two different values of the coupling $\beta$, hence at different distances in lattice units.

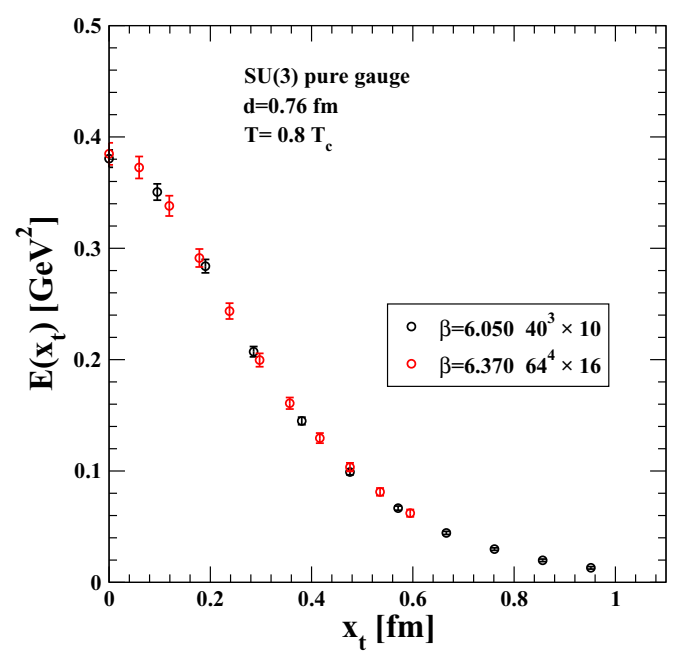

(a)

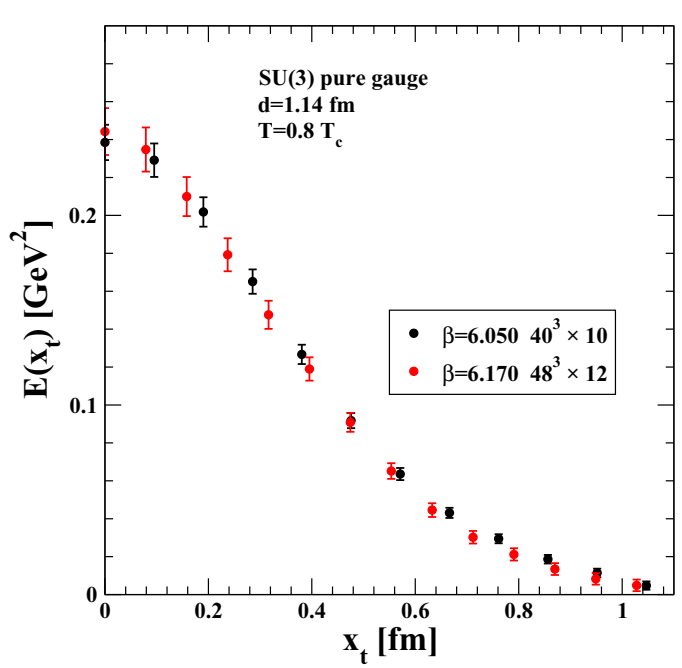

(b)

Figure 3. (a) The longitudinal chromoelectric field $E_{l}$ (in physical units) versus the distance $x_{t}$ (in physical units) from the axis connecting the static sources placed at physical distance $d=0.76 \mathrm{fm}$ at $T=0.8 T_{c}$. Black open circles refer to $\beta=6.050$ on a $40^{3} \times 10$ lattice, red open circles refer to $\beta=6.370$ on a $64^{3} \times 16$ lattice. (b) The longitudinal chromoelectric field $E_{l}$ (in physical units) versus the distance $x_{t}$ (in physical units) from the axis connecting the static sources placed at physical distance $d=1.14 \mathrm{fm}$ at $T=0.8 T_{c}$. Black open circles refer to $\beta=6.050$ on a $40^{3} \times 10$ lattice, red open circles refer to $\beta=6.170$ on a $48^{3} \times 12$ lattice.

In Fig. 3 we present the outcome of this test for two different values of the physical distance between the static quark-antiquark pair. Fig. 3(a) shows the (smeared) chromoelectric field versus the transverse distance $x_{t}$ in physical units, when the sources are placed at distance $8 a$ and $10 a$ at $\beta=6.050$ and $\beta=6.195$, respectively, that, in both cases, corresponds to a distance roughly equal to $0.76 \mathrm{fm}$ in physical units. Fig. 3(b) shows the (smeared) chromoelectric field versus the transverse distance $x_{t}$ in physical units, when the sources are placed at distance $12 a$ and $14 a$ at $\beta=6.050$ and $\beta=6.170$, respectively, that, in both cases, corresponds to a distance roughly equal to $1.14 \mathrm{fm}$ in physical units. In both cases an almost perfect scaling can be observed, thus making us confident that, for the observable of interest in this work, the continuum scaling is reached (at least) for $\beta=6.050$. Another hint from the results shown in Fig. 3 is that our use of the smearing procedure is robust: had the smearing procedure badly corrupted the physical signal for the chromoelectric field, it would have been quite unlikely to obtain such a nice scaling. 


\subsection{Flux tubes across deconfinement}

We studied the behavior of the flux tubes across deconfinement. In particular we measured the transverse shape of the dominant component of the field strength tensor, i.e. $E_{l}\left(x_{t}\right)$, having numerically checked, at least for the temperature $T=0.8 T_{c}$, that the other components are zero within statistical uncertainties (see Fig. 4). In Fig. 5 we present the results obtained for the chromoelectric longitudi-

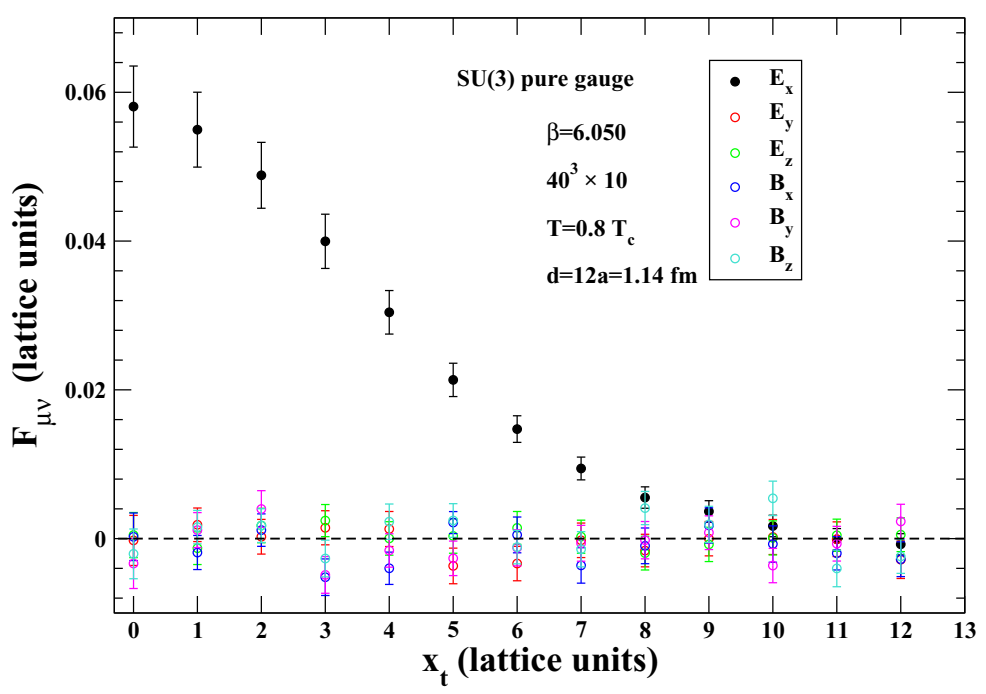

Figure 4. The chromoelectromagnetic field (Eq. (3)) components at $T=0.8 T_{c}$ for quark-antiquark at distance $d=12 a=1.14 \mathrm{fm}$.

nal field $E_{l}\left(x_{t}\right)$ in correspondence of temperatures $0.8 T_{c} \leq T \leq 1.5 T_{c}$ with the static quark-antiquark sources placed apart at distances $0.76 \mathrm{fm} \leq d \leq 1.33 \mathrm{fm}$. The data for $E_{l}\left(x_{t}\right)$ are well fitted for all temperatures $T$ and all distances $d$ by using a functional form introduced long ago [29] for describing flux tubes in ordinary superconductivity and recently proposed [14-16] in order to describe the transverse distribution of the chromoelectric flux tube:

$$
E_{l}\left(x_{t}\right)=\frac{\phi}{2 \pi} \frac{\mu^{2}}{\alpha} \frac{K_{0}\left[\left(\mu^{2} x_{t}^{2}+\alpha^{2}\right)^{1 / 2}\right]}{K_{1}[\alpha]} .
$$

In Eq. (4) $K_{n}$ is the modified Bessel function of order $n, \phi$ is the external flux, $\mu=1 / \lambda$ with $\lambda$ the London penetration length, and $\alpha=\xi_{v} / \lambda$ with $\xi_{v}$ a variational core radius parameter. The results in Fig. 5 suggest that the flux tube shape survives across the deconfinement phase transition up to $T=$ $1.5 T_{c}$, even though the strength of the field collapses across the phase transition. By using the fit Eq. (4) to the numerical data for the chromoelectric longitudinal field $E_{l}\left(x_{t}\right)$ displayed in Fig. 5 it is possible to evaluate the mean square root width of the chromoelectric flux tubes,

$$
\sqrt{w^{2}}=\sqrt{\frac{\int d^{2} x_{t} x_{t}^{2} E_{l}\left(x_{t}\right)}{\int d^{2} x_{t} E_{l}\left(x_{t}\right)}}=\sqrt{\frac{2 \alpha}{\mu^{2}} \frac{K_{2}(\alpha)}{K_{1}(\alpha)}},
$$




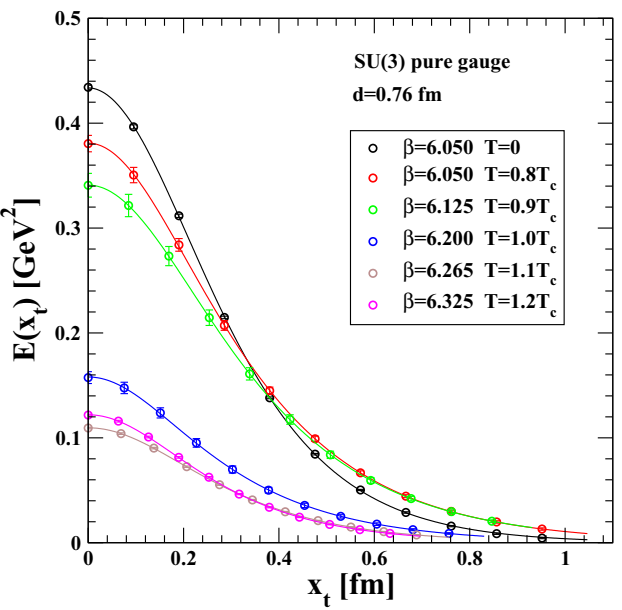

(a)

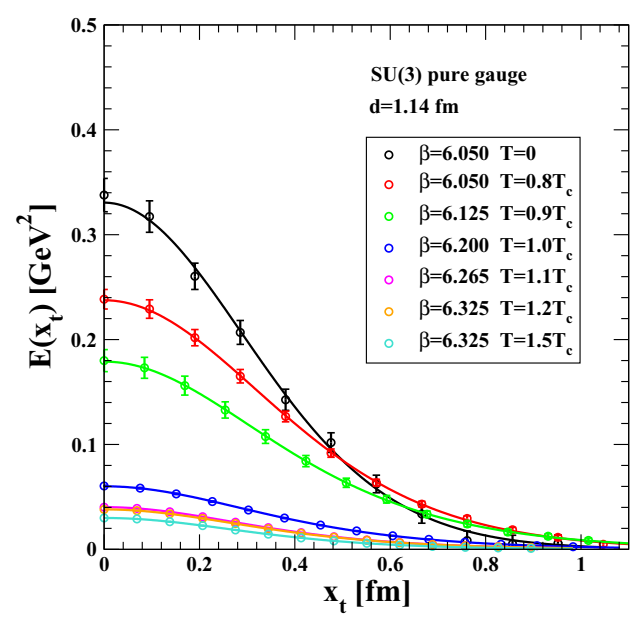

(c)

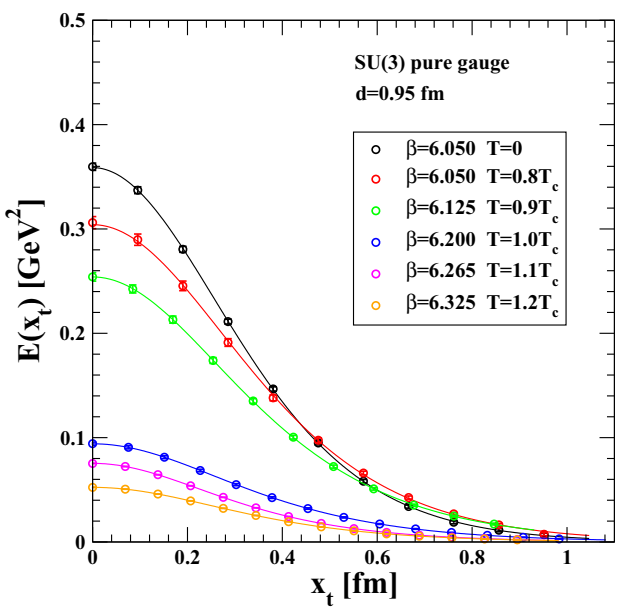

(b)

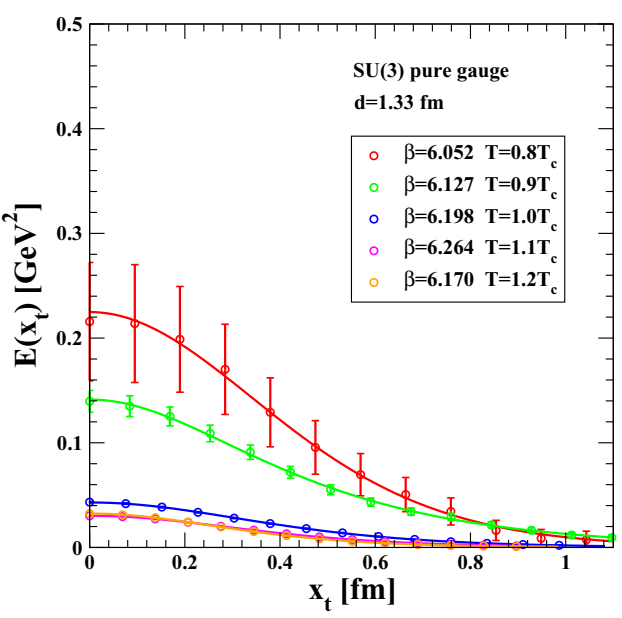

(d)

Figure 5. Behavior of the longitudinal chromoelectric field $E_{l}$ (in physical units) versus the distance $x_{t}$ (in physical units) from the axis connecting the static sources, at fixed value of the physical distance between the sources and in correspondence of several values of temperature across deconfinement.

and the square root of the energy per unit length, normalized to the flux $\phi$,

$$
\frac{\sqrt{\varepsilon}}{\phi}=\frac{1}{\phi} \sqrt{\int d^{2} x_{t} \frac{E_{l}^{2}\left(x_{t}\right)}{2}}=\sqrt{\frac{\mu^{2}}{8 \pi}\left(1-\left(\frac{K_{0}(\alpha)}{K_{1}(\alpha)}\right)^{2}\right)} .
$$

We can see that the flux $\phi$ drops down across deconfinement (Fig. 6(a)) while the penetration length $\lambda$ remains almost constant (Fig. 6(b)), as well as the mean square root width (Eq. (5)) of the flux tube and the square root of the energy per unit length (Eq. (6)). 


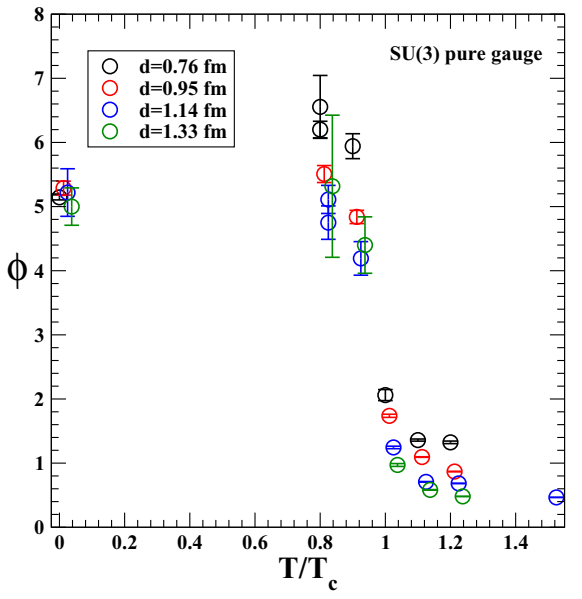

(a)

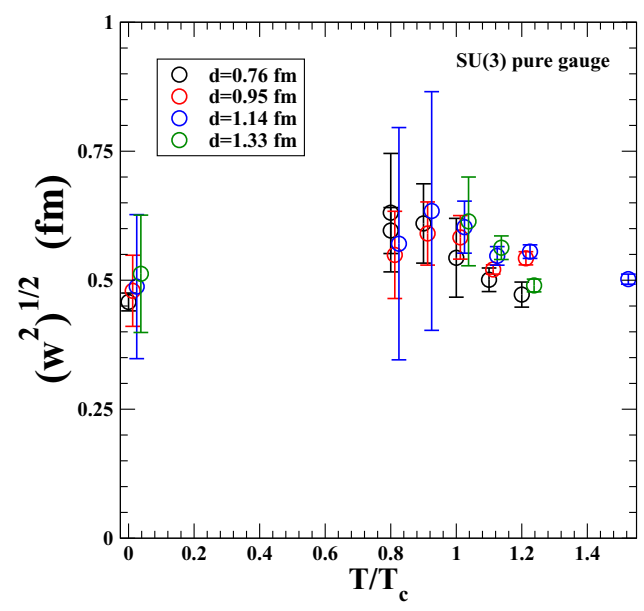

(c)

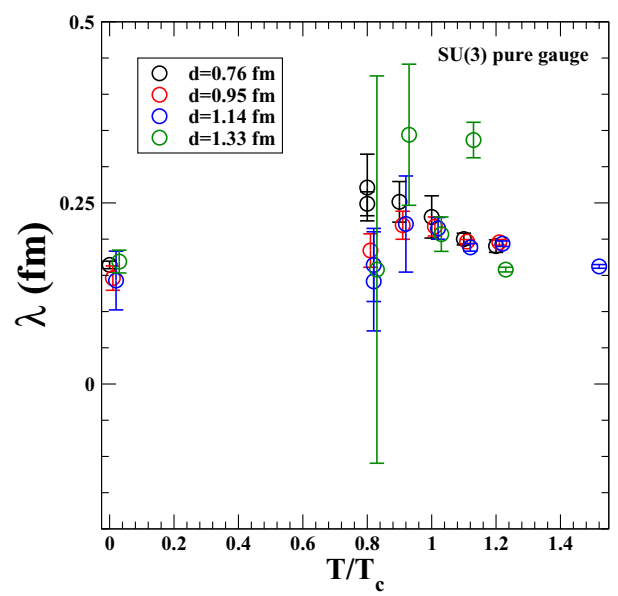

(b)

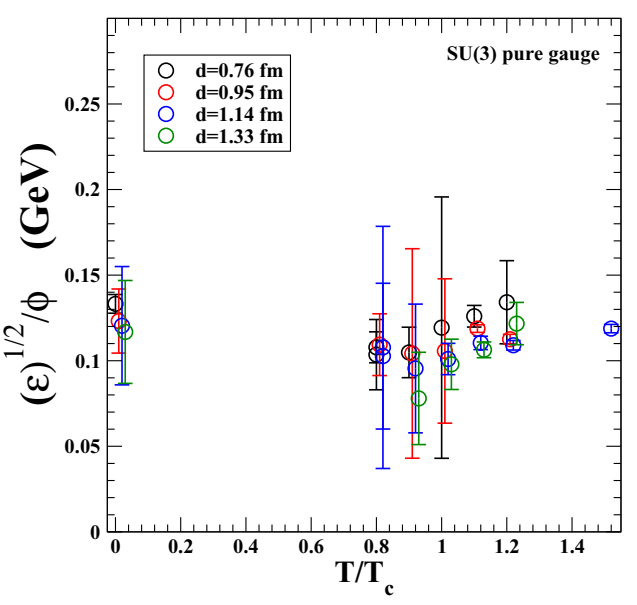

(d)

Figure 6. (a) The flux $\phi$ (Eq. (4)) versus $T / T_{c}$ for several values of the distance $d$ between the quark-antiquark sources. (b) The penetration length $\lambda=1 / \mu$ (Eq. (4)). (c) The root mean width of the flux tube (Eq. (5)). (d) The energy per unit length in the flux tube (Eq. (6)). Data point abscissas have been slightly shifted for readability.

\section{Conclusions}

We presented new preliminary results in studying the flux tube produced by a quark-antiquark pair in the case of SU(3) pure gauge theory across the deconfinement phase transition. We have seen that, at least for $T=0.8 T_{c}$, only the chromoelectric longitudinal field contributes to the field inside the flux tube and the other components are zero within statistical uncertainties. The shape of the chromoelectric longitudinal field can be well described using a functional form derived from the the ordinary superconductivity [29]. Noticeably the flux tube shape seems to survive across deconfinement up to $T=1.5 T_{c}$, even though the strength of the field collapses across the phase transition. We plan to 
check the stability of our results against the change of the smoothing procedure used to get rid of the large fluctuations at the scale of the lattice spacing. We also plan to extend our study in pure SU(3) gauge theory to the more realistic case of $(2+1)$-flavors QCD.

\section{Acknowledgments}

This investigation was in part based on the MILC collaboration's public lattice gauge theory code. See http://physics.utah.edu/ detar/milc.html. Numerical calculations have been made possible through a CINECA-INFN agreement, providing access to resources on GALILEO and MARCONI at CINECA.

\section{References}

[1] M. Fukugita, T. Niuya, Phys. Lett. B132, 374 (1983)

[2] J.E. Kiskis, K. Sparks, Phys. Rev. D30, 1326 (1984)

[3] J.W. Flower, S.W. Otto, Phys. Lett. B160, 128 (1985)

[4] J. Wosiek, R.W. Haymaker, Phys. Rev. D36, 3297 (1987)

[5] A. Di Giacomo, M. Maggiore, S. Olejnik, Nucl. Phys. B347, 441 (1990)

[6] P. Cea, L. Cosmai, Nucl. Phys. Proc. Suppl. 30, 572 (1993)

[7] Y. Matsubara, S. Ejiri, T. Suzuki, Nucl. Phys. Proc. Suppl. 34, 176 (1994), hep-lat/9311061

[8] P. Cea, L. Cosmai, Phys. Lett. B349, 343 (1995), hep-lat/9404017

[9] P. Cea, L. Cosmai, Phys. Rev. D52, 5152 (1995), hep-lat/9504008

[10] G.S. Bali, K. Schilling, C. Schlichter, Phys. Rev. D51, 5165 (1995), hep-lat/9409005

[11] R.W. Haymaker, T. Matsuki, Phys. Rev. D75, 014501 (2007), hep-lat/0505019

[12] A. D’Alessandro, M. D'Elia, L. Tagliacozzo, Nucl.Phys. B774, 168 (2007), hep-lat/0607014

[13] M.S. Cardaci, P. Cea, L. Cosmai, R. Falcone, A. Papa, Phys.Rev. D83, 014502 (2011), 1011.5803

[14] P. Cea, L. Cosmai, A. Papa, Phys.Rev. D86, 054501 (2012), 1208. 1362

[15] P. Cea, L. Cosmai, F. Cuteri, A. Papa, Phys. Rev. D89, 094505 (2014), 1404.1172

[16] P. Cea, L. Cosmai, F. Cuteri, A. Papa, PoS LATTICE2014, 350 (2014), 1410. 4394

[17] N. Cardoso, M. Cardoso, P. Bicudo, Phys. Rev. D88, 054504 (2013), 1302 . 3633

[18] M. Caselle, M. Panero, R. Pellegrini, D. Vadacchino, JHEP 01, 105 (2015), 1406. 5127

[19] P. Bicudo, N. Cardoso, M. Cardoso (2017), 1702.03454

[20] C. Bonati, S. Cali, M. D’Elia, F. Negro, A. Rucci, F. Sanfilippo, Flux tubes in $N f=2+1 Q C D$ with external fields, in Proceedings, 35th International Symposium on Lattice Field Theory (Lattice2017): Granada, Spain, to appear in EPJ Web Conf.

[21] P. Cea, L. Cosmai, F. Cuteri, A. Papa, Phys. Rev. D95, 114511 (2017), 1702.06437

[22] P. Cea, L. Cosmai, F. Cuteri, A. Papa, JHEP 06, 033 (2016), 1511.01783

[23] D.S. Kuzmenko, Y.A. Simonov, Phys. Lett. B494, 81 (2000), hep-ph/0006192

[24] M. Baker, Phys. Rev. D93, 054012 (2016), 1512.02705

[25] R.G. Edwards, U.M. Heller, T.R. Klassen, Nucl. Phys. B517, 377 (1998), hep-lat/9711003

[26] http://physics.utah.edu/ detar/milc.html

[27] A. Hasenfratz, F. Knechtli, Phys. Rev. D64, 034504 (2001), hep-lat/0103029

[28] M. Falcioni, M. Paciello, G. Parisi, B. Taglienti, Nuclear Physics B 251, 624 (1985)

[29] J.R. Clem, Journal of Low Temperature Physics 18, 427 (1975), 10.1007/BF00116134 